\title{
Hot and Cool Forms of Inhibitory Control and Externalizing Behavior in Children of Mothers who Smoked during Pregnancy: An Exploratory Study
}

\author{
Stephan C. J. Huijbregts - Alison J. Warren • \\ Leo M. J. de Sonneville • Hanna Swaab-Barneveld
}

Published online: 9 October 2007

(C) Springer Science + Business Media, LLC 2007

\begin{abstract}
This study examined whether children exposed to prenatal smoking show deficits in "hot" and/or "cool" executive functioning (EF). Hot EF is involved in regulation of affect and motivation, whereas cool EF is involved in handling abstract, decontextualized problems. Forty 7 to 9-year-old children (15 exposed to prenatal smoking, 25 non-exposed) performed two computerized tasks. The Sustained Attention Dots (SA-Dots) Task (as a measure of "cool" inhibitory control) requires 400 non-dominant hand and 200 dominant hand responses. Inhibitory control of the prepotent response is required for dominant hand responses. The Delay Frustration Task (DeFT) (as a measure of "hot" inhibitory control) consists of 55 simple maths exercises. On a number of trials delays are introduced before the next question appears on the screen. The extent of responsebutton pressing during delays indicates frustration-induced inhibitory control. Prenatally exposed children showed poorer inhibitory control in the DeFT than non-exposed children. A dose-response relationship was also observed. In addition, prenatally exposed children had significantly higher (dose-dependent) conduct problem- and hyperactivityinattention scores. There were no significant group differences in inhibitory control scores from the SA-Dots. These
\end{abstract}

S. C. J. Huijbregts $(\varangle) \cdot$ L. M. J. de Sonneville •

H. Swaab-Barneveld

Department of Clinical Child and Adolescent Studies

Neurodevelopmental Disorders, Faculty of Social Sciences,

Leiden University,

P.O. Box 9555, 2300 RB Leiden, The Netherlands

e-mail: SHuijbregts@fsw.leidenuniv.nl

\section{A. J. Warren}

School of Psychology, University of Southampton,

Southampton, UK results indicate that children exposed to prenatal smoking are at higher risk of hot but not cool executive function deficits.

Keywords Prenatal smoking $\cdot$ Hot and cool executive functioning · Inhibitory control Conduct problems . Hyperactivity

\section{Introduction}

Prenatal tobacco exposure has consistently been associated with children's externalizing behavior (e.g. Button et al. 2005; Huijbregts et al. 2007, in press; Kotimaa et al. 2003; Mick et al. 2002; Wakschlag et al. 2006). In contrast, studies into associations between prenatal tobacco exposure and children's cognitive functioning show very mixed results (e.g. Batstra et al. 2003; Fried et al. 1998, 2003 versus Breslau et al. 2005; Huijbregts et al. 2006; Trasti et al. 1999). This is surprising because externalizing behavior problems often co-occur with cognitive difficulties.

\section{Cognition in ADHD, ODD/CD, and ADHD+ODD/CD}

Cognition has been widely studied in ADHD (for reviews, see Barkley 1997; Castellanos et al. 2006; Sergeant et al. 2003; Wilcutt et al. 2005). This research has shown, among others, problems with inhibitory control, working memory, vigilance, reward sensitivity (delay aversion), and time processing and currently focuses on different cognitive endophenotypes of ADHD (i.e. biologically based phenotypes that carry genetic loadings and index liability to disease; see Castellanos and Tannock 2002). Oppositional Defiant Disorder (ODD), Conduct Disorder (CD) and high levels of physical aggression have also been associated with cognitive difficulties such as language-based verbal skills 
and executive function (EF) (Henry and Moffitt 1997; Hill 2002; Séguin et al. 1999). Controversy still exists about whether verbal and, particularly, EF deficits are present in ODD/CD without comorbid ADHD (Pennington and Ozonoff 1996). Since Pennington and Ozonoff's review on $\mathrm{EF}$ in different forms of developmental psychopathology, an increasing number of studies have taken into account the high comorbidity of ODD/CD and ADHD. This has resulted in reports of both qualitative and quantitative differences in EF-dysfunction between disorders. For example, Van Goozen and colleagues (2004) reported the absence of problems with Working Memory (WM) and planning in a group of children with Oppositional Defiant Disorder (ODD) and a group with combined ODD/ADHD compared to healthy controls. Both are executive functions that have frequently been reported to be deficient in ADHD. Oosterlaan et al. (2005) compared ADHD, ODD/CD, and ADHD+ODD/ $\mathrm{CD}$ groups and found that $\mathrm{WM}$ and planning deficits were specific to ADHD (and explained by ADHD in the combined disorder). Distinguishing $\mathrm{ADHD}$ and $\mathrm{ODD} / \mathrm{CD}$ is more difficult when it concerns inhibitory control (Oosterlaan et al. 1998). Inhibition has been defined and operationalized in a number of different ways, e.g. as stopping an ongoing response, interference control and inhibition of prepotent responses (Barkley 1997), and studies have generally not included tasks measuring such different forms of inhibition. Furthermore, inhibition has been listed as one of the executive functions (e.g. Pennington and Ozonoff 1996), but has also been described as an important cognitive ability underlying many different executive functions (Zelazo et al. 1997). Lack of inhibitory control has been proposed as the central deficit in both ADHD (Barkley 1997) and ODD/CD/ physical aggression (e.g. Lau et al. 1995), but is not necessarily similarly dysfunctional in both types of disorders. Nigg (2003) has suggested that ADHD is predominantly associated with dysfunctional executive inhibition, whereas conduct problems are predominantly associated with dysfunctional motivational inhibition. Nonetheless, a 'motivational' pathway (or endophenotype) to cognitive deficits (e.g. altered reward sensitivity) in ADHD has also been identified (Solanto et al. 2001; Sonuga-Barke 2002). ODD/CD and ADHD may be distinguished more easily on other aspects of emotional or social information processing. As a starting point it may therefore be good to use a theoretical framework that, in addition to motivation, accommodates more different aspects of emotional or social information processing. Such a model has been proposed by Zelazo and Müller (2002).

\section{Hot and Cool Aspects of Executive Functioning}

Zelazo and Müller (2002) made a distinction between the 'cool' aspects of EF more associated with dorsolateral regions of the prefrontal cortex (PFC) and the 'hot' aspects more associated with ventral and medial regions. Cool EF is elicited by abstract, decontextualized problems, whereas hot EF is elicited by problems that involve the regulation of affect and motivation (i.e., the regulation of basic limbic system functions). Neuro-anatomical evidence for a distinction between hot and cool executive functions stems from research into risky ('hot') decision making versus ambiguous ('cool') decision making (Krain et al. 2006). The authors performed a meta-analysis of studies measuring brain activity during risky decision making (involving low probabilities of high rewards and high probabilities of low rewards) and/or ambiguous decision making (where the probability of a specific outcome is unknown or close to chance and choices do not differ in reward value), and found that there were significant differences between risky and ambiguous decision-making in regions of the orbitofrontal cortex (OFC: more activity in risky than in ambiguous decision-making), dorsolateral prefrontal cortex (DLPFC: more activity in ambiguous than in risky decision making) and anterior cingulate cortex (ACC: more activity in ambiguous decision-making than in risky decision-making in caudal regions, and more activity in risky than in ambiguous decision-making in rostral regions).

Zelazo and Müller use a problem-solving framework to discuss EF. Each stage of problem-solving (problem representation, planning, execution, and evaluation) is subserved by EF, which can take both hot and cool forms. They provide examples of each for every problem solving stage. For example, for the problem representation stage cool EF could be measured by asking participants to rerepresent test items in different ways (but not act upon the re-representation). Hot measures of problem representation would include most tests of Theory of Mind, where participants are required to represent something (e.g. feelings, intentions) from multiple points of view. For the planning stage, tower tasks that require children to describe their plans without actually executing them are put forward as an example of 'cool' EF, whilst strategic deception, where participants are shown two pre-formulated plans, one deceptive and one not, and are asked to select the one that would trick the other person, was offered as an example of 'hot' EF. In the execution stage, card sorting (i.e. select according to two different rules/principles) was given as an example of cool EF, and delay of gratification as an example of hot EF (e.g. choosing between a small reward immediately or a larger reward later (prudence) or between a reward for self now or a reward for self and other later (altruism)). Finally, in the evaluation phase error detection and error correction in the context of monitoring progress toward a goal are provided as examples of cool EF and error detection and correction in the context of extinction as examples of hot EF (i.e. after a certain amount of responding, it can be shown that the reward supply has 
been exhausted, a motivational input that might lead to response extinction). In the problem-solving framework inhibitory control is considered to underlie EF in all information processing stages and consequently has its hot and cool variants as well. Thus, when inhibitory control involves emotion, affect, and/or motivation, it may be classified as a hot EF, whereas inhibitory control could be classified as cool EF when it is required in an "abstract" or "neutral" context.

\section{Neurobiology of Prenatal Tobacco Exposure} and Externalizing Behavior

There is increasing evidence that maternal prenatal smoking is more strongly associated with $\mathrm{ODD} / \mathrm{CD}$ and with the cooccurrence of ODD/CD and ADHD than with ADHD alone (Huijbregts et al. 2007; Nigg and Breslau 2007; Wakschlag et al. 2006). This evidence alone might be enough to suggest that children prenatally exposed to tobacco might specifically show cognitive dysfunction when emotion is involved, specifically when tasks involve inhibition. Further indirect support for such a hypothesis comes from neurobiological evidence showing similar or closely related effects on the central and peripheral nervous system associated with prenatal smoking and with antisocial/ disruptive behavior. Antisocial and disruptive behaviors have convincingly been associated with changes in the functioning of the autonomic nervous system (ANS) and the hypothalamic-pituitary-adrenal (HPA) axis. Both have very important roles in stress regulation. ANS-functioning is generally measured by (changes in) heart rate (HR), which reflects sympathetic and parasympathetic nervous system activity, and (changes in) skin conductance (SC), which reflects only sympathetic nervous system activity. HPAfunctioning is generally measured by (changes in) the adrenal stress hormone cortisol. Resting HR, basal SC and basal cortisol levels were all shown to be lower in children with disruptive behavior disorders. In addition, reduced SC reactivity and blunted or absent cortisol responses to stress have consistently been reported (for reviews, see Ortiz and Raine 2004; Van Goozen et al. 2007). These findings have lead to the fearlessness theory of antisocial behavior (Raine 1993), which claims that low levels of arousal are markers of low levels of fear and the stimulation-seeking theory (Zuckerman 1979), which claims that low arousal represents an aversive physiological state and that individuals with tonically low arousal seek out stimulation to raise their arousal to optimal or normal level.

Antisocial/disruptive behavior has also been associated with neurotransmitter abnormalities, for example with impairments in the serotonergic (5-HT) system. The 5-HT system interacts with the HPA-system at several levels. For example, activation of (postsynaptic) 5-HT receptors in the hypothalamus stimulates HPA axis activity and causes elevation in cortisol, while a disruption of 5-HT neurotransmission by tryptophan depletion lowers HPA axis functioning (blunts cortisol-elevating effect of stressor). Glucocorticoids also modulate 5-HT receptor binding/sensitivity (see Van Goozen et al. 2007). Importantly, the ANS/HPA abnormalities in children with conduct problems have not been replicated in children with ADHD only (Herpertz et al. 2001; Snoek et al. 2004; Waschbusch 2002).

Animal studies investigating the consequences of prenatal nicotine exposure have shown an upregulation of nicotinic cholinergic receptor binding sites as well as persistent deficits in cerebrocortical choline acetyltransferase activity and hemicholium-3 binding to the presynaptic choline transporter, which is indicative of cholinergic hypoactivity (Seidler et al. 1992; Slotkin et al. 2006). These studies also show changes in noradrenergic responsiveness following prenatal nicotine exposure. The most important neurotransmitters of the ANS are acetylocholine (parasympathetic) and norepinephrine (sympathetic). In line with the chronic underarousal theories of antisocial/disruptive behavior, it may be hypothesized that, without continued nicotine exposure, the increased number of cholinergic receptor binding sites will be understimulated, possibly resulting in stimulation-seeking and fearlessness. Furthermore, abnormalities in serotonergic neuromodulation following prenatal nicotine exposure have been indicated (suppression of the $5 \mathrm{HT}_{1 \mathrm{~A}}$ receptor subtype and upregulation of $5 \mathrm{HT}_{2}$ receptor subtype) (Seidler et al. 1992; Slotkin et al. 2006). We had already stressed the interactions between 5HT and HPAfunctioning. The commonalities in the neurobiology of antisocial/disruptive behavior and the neurobiology of prenatal smoking are striking. Moreover, factors affecting the same neurobiological system are more likely to reinforce each other's impact (Moffitt et al. 2005). A number of studies have already reported abnormalities in ANS/HPAfunctioning and levels of arousal in children of mothers who smoked during pregnancy (Browne et al. 2000; Horne et al. 2004; McDonald et al. 2006; Ramsay et al. 1996).

\section{The Present Study}

Taken together, the strong associations between prenatal smoking and offspring conduct problems, the specific affect-mediated ('hot') cognitive deficits supposedly associated with conduct problems, and the common neurobiology of prenatal nicotine exposure and conduct problems that particularly affects behavior and cognition under stressful conditions (i.e. behavior and cognition that is affect-mediated or 'hot'), suggest that it would be useful to investigate aspects of hot cognition in children exposed to prenatal smoking (see also Raine 2002). Studies into associations between prenatal tobacco exposure and cogni- 
tion have generally focused on academic achievement or rather broad cognitive constructs such as intelligence, verbal ability and executive functions (e.g. Batstra et al. 2003; Breslau et al. 2005; Huijbregts et al. 2006; Trasti et al. 1999), and have not yet used a distinction between hot and cool aspects of cognition. There are many possible causes for the inconsistent results which will be discussed later, but in the present study we will contrast hot and cool aspects of cognition, more specifically hot and cool forms of inhibitory control because a lack thereof has been hypothesized to be central to the behavior problems displayed by children of mothers who smoked during pregnancy. It is expected that children of mothers who smoked will particularly display problems with hot inhibitory control. Since a number of shared neural structures and neuromodulatory mechanisms are involved in cool and hot EF it cannot be ruled out that children prenatally exposed to nicotine will also have problems with cool inhibitory control but it may be hypothesized that these, if present, will be of a less serious nature.

\section{Method}

\section{Participants}

Forty children (16 boys, 24 girls; all of Caucasian ethnicity) attending two primary schools in Southampton (UK) participated in the study: 25 children from mothers who had not smoked during pregnancy ( 9 boys, 16 girls), 15 from mothers who had smoked during pregnancy ( 7 boys, 8 girls). Of these mothers, 6 smoked 1-9 cigarettes/day and 9 smoked $\geq 10$ cigarettes/day (prenatal smoking was established through self-report by the mothers; although there is a risk for a social desirability bias, several studies have indicated a relatively strong association between retrospective self-report and blood/urine cotinine-levels (i.e. the main nicotine metabolite), e.g. Law et al. 2003; Pickett et al. 2005). Mean age of the participants was 7.98 (range 7.0-9.0, $\mathrm{SD}=0.68$ ). Informed consent was obtained from all children and their parents/caretakers and from the school boards. The study was approved by the University of Southampton-School of Psychology Ethics Committee.

Tasks and Measures

\section{Sustained Attention Dots Task}

The Sustained Attention Dots task (SA-Dots, De Sonneville 1999) is a computerized task consisting of 600 presentations of 3-, 4-, and 5-dot patterns (random configurations). The 3, 4, or 5 dots are each presented 200 times.
Participants use both hands for responding. They must press the mouse key of the non-dominant hand (the "nokey') when 3 or 5 dots appear on the screen and the mouse key of the dominant hand (the 'yes-key') when 4 dots appear. A response has to be given between 250 and $6,000 \mathrm{~ms}$ after a signal. The fixed post-response interval is $250 \mathrm{~ms}$. The program provides auditory feedback after an error. Since a response with the non-dominant hand is required twice as often as a response with the dominant hand, a response with the non-dominant hand becomes the prepotent response. Thus, inhibitory control is required when four dots appear. The less participants are capable of inhibiting biased response tendencies, the more misses (i.e. pressing the 'no-key' when four dots appear) they will produce relative to false alarms (i.e. pressing the 'yes-key' when three or five dots appear). The Bias score is the difference between number of misses and the mean of the number of false alarms when three dots appeared and when five dots appeared (see Huijbregts et al. 2002a). A second measure used to assess the quality of inhibitory control is post-error slowing. Post-error slowing is usually considered a strategic adjustment based on performance monitoring (Klein et al. 2007; Wiersema et al. 2007). In order to be able to slow down after an error inhibitory control is required. In order to control for general response speed, the difference between mean RT of correct responses after errors and overall mean RT of correct responses was selected as measure for post-error slowing (RTae).

\section{Delay Frustration Task}

The Delay Frustration Task (DeFT, Bitsakou et al. 2006) is a task where participants are presented with a series of simple maths questions (only additions in the children's version) on a computer screen. Four possible solutions are displayed on the screen together with each question. Participants are asked to select the correct answer by pressing one of four buttons on a response box. The next question is presented as soon as a response has been recorded. However, on 16 out of 55 trials access to the next trial is delayed. On 8 out of the 16 trails in the post-response delay condition, a short delay (2-10 s) occurs, whilst on the remaining eight trials a delay of $20 \mathrm{~s}$ occurs. During the $20 \mathrm{~s}$ delay periods, the number (NP) and duration (TP) of responses made on a response key are recorded. These measures and their product (NP*TP) index delay frustration. In the instruction, participants are told that the computer shows signs of malfunctioning and that if the computer appears to not register their response, they should just wait until the next trial appears and do not have to respond again. It is considered to be indicative of inhibitory control if participants refrain from constantly pressing response box buttons 
during the delay periods. Practice sessions preceded both the SA-dots- and the DeFT test sessions, which were held in separate quiet rooms in both schools. Total test time per child was approximately $30 \mathrm{~min}$ (approximately $15 \mathrm{~min}$ for each task).

\section{Strengths and Difficulties Questionnaire (SDQ)}

The parent-teacher rated SDQ (Goodman 1997) is a short behavioral screening questionnaire of children aged 4 to 16 years, which can be completed within approximately $5 \mathrm{~min}$. For the present study, teacher ratings were obtained, which correlate highly with parent ratings (Goodman 1997). The SDQ consists of 25 items comprising five different subscales (five items each): emotional problems, conduct problems, hyperactivity/inattention, peer relationship problems, and prosocial behavior. The validity of this five-factor structure has been confirmed in large population samples (e.g. Goodman 2001). Each item is scored on a three-point scale: 'not true', 'somewhat true', or 'certainly true'. 'Somewhat true' is always scored as 1 , with the scoring of 'not true' and 'certainly true' varying with each item, being equal to either 0 or 2 . A higher score indicates greater problems except for prosocial behavior, where a higher score indicates more positive behavior. A total difficulties score (range 0-40) is obtained by summing the scores of the emotional problems-, conduct problems-, hyperactivity/inattention-, and peer relationship problems subscales. Of specific interest for the present study are the Conduct Problems (CP) scale, which has the following items: (1) often has temper tantrums or hot tempers; (2) generally obedient, usually does what adults request; (3) often fights with other children or bullies them; (4) often lies or cheats; and (5) steals from home, school, or elsewhere, and the Hyperactivity/Inattention (HI) scale, containing the following items: (1) restless, overactive, cannot stay still for long; (2) constantly fidgeting or squirming; (3) easily distracted, concentration wanders; (4) thinks things out before acting; and (5) sees tasks through to the end, good attention span. Examples of items from the other scales are: "Picked on or bullied by other children" (peer relationship problems); "Many worries, often seems worried" (emotional problems); and "Helpful if someone is hurt, upset or feeling ill" (prosocial behavior). The SDQ has good reliability, whether it concerns internal consistency of the items (mean Cronbach's $\alpha=0.73$ ), cross-informant correlations or test-retest reliability, and good criterion validity, whether it concerns its predictiveness of diagnosed psychiatric disorders, (mental health) service contact/use, or the association of its scores with those on other behavioral screening questionnaires (Bourdon et al. 2005; Goodman 2001; Goodman and Scott 1999).
Statistical Analysis

\section{Main analyses}

Because the number of children participating in this study was not large and the scores on the SA-Dots Task, the Delay Frustration Task, and the behavior scales of the Strengths and Difficulties Questionnaire were not normally distributed, we used nonparametric tests. Spearman rank order correlations were used to test whether scores on the Delay Frustration Task were related to scores on the SADots Task.

Mann-Whitney U tests were employed to compare children of non-smokers and children of mothers who smoked during pregnancy on cognitive and behavioral (SDQ) measures. In order to test for dose-dependency children of smoking mothers were further subdivided into those whose mothers smoked between one and nine cigarettes/day and those whose mothers smoked $\geq 10$ cigarettes/day. These two groups were compared to each other and to children of mothers who did not smoke using Kruskal-Wallis $H$-tests.

\section{Exploratory analyses}

Our final analyses were hierarchical linear regression analyses predicting those variables for which significant group differences were observed in Mann-Whitney $U$ tests from prenatal smoking and the sum of SDQ HyperactivityInattention (HI) and Conduct Problems (CP) scores. Maternal prenatal smoking was introduced in a first block followed by $\mathrm{CP}+\mathrm{HI}$ in a second block. These analyses should be considered exploratory because the sample is too small to reliably distinguish children with $\mathrm{HI}$ only or $\mathrm{CP}$ only. In order to determine whether $\mathrm{HI}$ or $\mathrm{CP}$ is more strongly associated with task performance than a combination of both of them, it would be necessary to have a HI only and CP only group as well (see Huijbregts et al. 2007; in press). Because the regression analyses are parametric tests, dependent variable scores were square root transformed in order to approach normal distributions. These exploratory analyses were performed because the relationship between prenatal smoking, externalizing behavior, and task performance can only be fully explored when all three variables are present in one analysis.

\section{Results}

Main analyses

Table 1 shows means (+SDs) of the smoking and nonsmoking groups for the subscales of the SDQ and for the 
Table 1 Mean scores (+SDs) for children with and without prenatal tobacco exposure on the strengths and difficulties questionnaire, the SA-dots task and the delay frustration task

\begin{tabular}{|c|c|c|c|c|c|c|c|c|}
\hline \multirow[t]{2}{*}{ Group } & \multirow[t]{2}{*}{ Number $(N)$} & \multicolumn{2}{|l|}{ SDQ } & \multicolumn{2}{|l|}{ SA-Dots } & \multicolumn{3}{|c|}{ Delay frustration task } \\
\hline & & $\mathrm{CP}$ & HI & RTae & Bias & $\mathrm{NP}$ & $\mathrm{TP}$ & NP*TP \\
\hline Overall & 40 & $0.68(1.2)$ & $2.8(2.5)$ & $477.2(507.1)$ & $9.0(8.9)$ & $0.40(.44)$ & $162.0(145.5)$ & $123.2(185.9)$ \\
\hline Not-Exposed & 25 & $0.48(1.2)$ & $2.2(2.2)$ & $473.6(594.1)$ & $9.0(10.3)$ & $0.29(.40)$ & $116.9(123.3)$ & $78.0(139.5)$ \\
\hline Exposed & 15 & $1.0(1.2)$ & $3.9(2.6)$ & $483.6(323.1)$ & $9.1(6.5)$ & $0.57(.46)$ & $234.1(153.0)$ & $195.5(229.6)$ \\
\hline
\end{tabular}

$S D Q$ : Strengths and Difficulties Questionnaire, $C P$ : Conduct Problems, HI: Hyperactivity-Inattention, SA-Dots: Sustained Attention-Dots; Bias and RTae are the inhibitory control measures from the SA-Dots task: RTae: RT correct after error - RT correct general; Bias: Number of errors on 4-dot presentations - mean number of errors on 3 and 5 dot presentations. $N P$, TP and $N P^{*} T P$ are the inhibitory control measures from the Delay Frustration Task: NP: mean number of response button presses during 20-s delays. TP: time response buttons were pressed during 20 -s delays.

outcome measures of the SA-Dots and the DeFT. Scores on the SA-Dots Task and the Delay Frustration Task did not correlate significantly: Bias $\times \mathrm{NP}: r=0.27, p=0.143$; Bias $\times$ TP: $r=0.27, p=0.139$; Bias $\times$ NP*TP: $r=0.26, p=0.150$; RTae $\times$ NP: $r=0.16, p=0.355$; RTae $\times$ TP: $r=0.13$, $p=0.453$; RTae $\times$ NP*TP: $r=0.20, p=0.267$. Despite the fact that scores on all different subscales of the SDQ were related to each other, only the scores on the hyperactivityinattention and conduct problems scales could be predicted by maternal prenatal smoking, as shown by the outcomes of a series of Mann-Whitney tests. Thus, prenatal smoking did not predict the score on the emotional problems scale: Mann-Whitney $U=183.5, z=-0.46, p=0.647$, the peer relationships problem scale: Mann-Whitney $U=185.0, z=-0.44, p=0.661$, or the prosocial behavior scale: Mann-Whitney $U=194, z=-0.17 p=0.865$. In contrast, children of mothers who had smoked during pregnancy had more conduct problems (Mann-Whitney

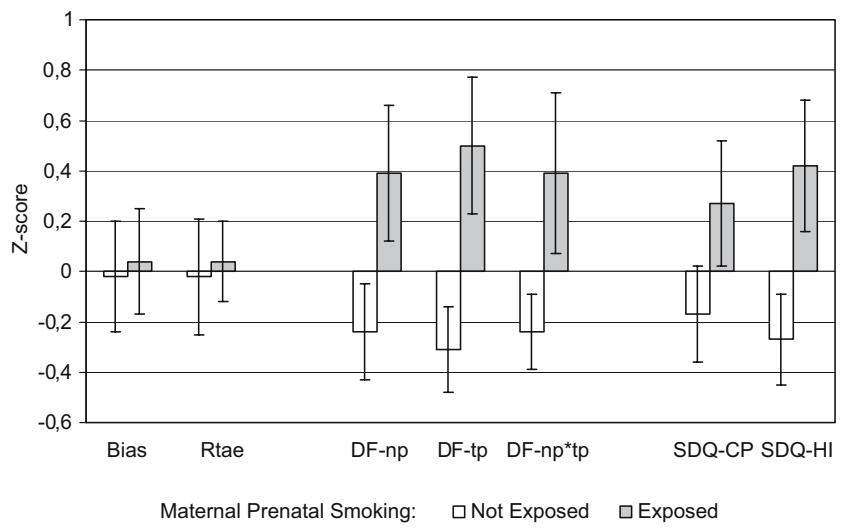

Fig. 1 Mean standardized scores on the SA-dots task, the Delay Frustration Task, and the Strengths and Difficulties Questionnaire for non-exposed vs exposed offspring. Bias and RTae are the inhibitory control measures from the SA-Dots task. $D F-n p$ (mean number of presses during $20 \mathrm{~s}$ intervals), $D F-t p$ (mean duration of presses during $20 \mathrm{~s}$ intervals), and $D F-n p^{*} t p$ (product of $D F-n p$ and $D F-t p)$ are the inhibitory control measures from the Delay Frustration Task. SDQ-CP: Strengths and Difficulties Questionnaire-Conduct Problems, SDQ-HI: Strengths and Difficulties QuestionnaireHyperactivity/Inattention
$U=134.5, z=-2.1, p=0.038)$ and higher hyperactivity/ inattention (Mann-Whitney $U=124.5, z=-2.0, p=0.041$ ) than children of mothers who had not smoked (mean ranks for conduct problems: 25.7 for exposed children and 18.0 for non-exposed children; for hyperactivity-inattention: 25.1 for exposed children and 18.4 for non-exposed children) (see also Fig. 1 for standardized CP and HIscores for non-exposed versus exposed children). Doseresponse relationships were observed when behavior of non-exposed, moderately exposed and heavily exposed offspring was compared with Kruskal-Wallis tests (conduct problems: mean ranks: $17.8,22.4$, and $26.8 ; \chi^{2}(2)=$ 5.7, $p=0.058$; hyperactivity/inattention: mean ranks 17.8 , 19.1, and 29.1; $\chi^{2}(2)=6.5, p=0.040$ ) (Fig. 2).

With respect to task performance, children of mothers who had smoked during pregnancy (mean rank=25.5) showed a higher response frequency (number of button presses: NP) during intervals in the Delay Frustration Task than children of non-smokers (mean rank=16.5) [MannWhitney $U=97, z=-2.4, p=0.016]$. They also showed a

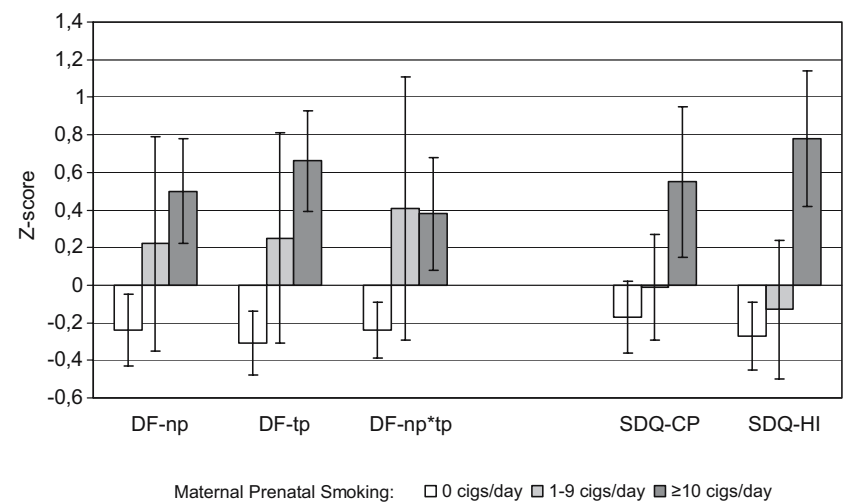

Fig. 2 Dose-dependency in associations between maternal prenatal smoking and scores on the Delay Frustration Task and the Strengths and Difficulties Questionnaire. $D F-n p$ (mean number of presses during $20 \mathrm{~s}$ intervals), $D F$-t $p$ (duration of presses during $20 \mathrm{~s}$ intervals), and $D F-n p^{*} t p$ (product of $D F-N P$ and $D F-T P$ ) are the inhibitory control measures from the delay frustration task. $S D Q-C P$ : Strengths and Difficulties Questionnaire - Conduct Problems, SDQ-HI: Strengths and Difficulties Questionnaire-Hyperactivity/Inattention 
longer response duration during intervals (time of button pressing: TP) (mean rank=26.4) than children of nonsmokers (mean rank=16.0) [Mann-Whitney $U=84$, $z=-2.8, p=0.005]$ (Table 1; Fig. 1). A dose response relation was shown by Kruskal-Wallis tests: NP: 0 cigs/ day: mean rank $=16.5 ; 1-9$ cigs/day: mean $r a n k=23.0 ; \geq 10$ cigs/day: mean rank $=27.2 ; \chi^{2}(2)=6.2, p=0.044 ; \mathrm{TP}: 0 \mathrm{cigs} /$ day: mean rank $=16.0 ; 1-9$ cigs/day: mean rank $=23.2 ;=10$ cigs/day: mean rank=28.6, $\chi^{2}(2)=8.5, p=0.014$ (Fig. 2). For frequency and duration combined (NP*TP), a Mann-Whitney test also showed that children of smokers (mean rank=26.0) showed less restraint during delays than children of nonsmokers (mean rank=16.3): $U=90, z=-2.6, p=0.009$. A dose-response relationship, indicating less inhibitory control during delays with an increase in prenatal smoking, was also observed (mean ranks for children of non-smokers, moderate smokers, and heavy smokers were 16.3, 22.8, and 28.1, respectively): $\chi^{2}(2)=7.5, p=0.023$ (Fig. 2).

There were no significant differences between exposed and non-exposed offspring in inhibitory control scores from the Sustained Attention Task: Bias: $U=130.5, z=-1.1$, $p=0.255$; RTae: $z=-0.03, p=0.988$; Dose response: Bias: $\chi^{2}(2)=3.1, p=0.209$; RTae: $\chi^{2}(2)=1.4, p=0.502$.

\section{Exploratory analyses}

An important question that remains is whether the levels of $\mathrm{CP}$ and $\mathrm{HI}$ explain associations of prenatal smoking with DeFT-performance. As expected $\mathrm{HI}$ and $\mathrm{CP}$ were significantly related (Spearman's rho $=0.57, p<0.001$ ). Because not enough children with $\mathrm{CP}$ only or HI only could be distinguished in our sample, the sum of the SDQ CP- and $\mathrm{HI}$-scores was used in subsequent regression analyses.

Hierarchical linear regression analyses introducing prenatal smoking in a first block and $\mathrm{CP}+\mathrm{HI}$ in a second block to predict DeFT-scores NP, TP and NP*TP showed significant regression models for each of the DVs: NP: $F(2,32)=3.9, p=0.029$; TP: $F(2,32)=7.5, p=0.002$; and NP*TP: $F(2,32)=3.8, p=0.039$. In none of these models prenatal smoking remained a significant predictor when $\mathrm{CP}+\mathrm{HI}$ was introduced: NP: $\beta=0.268(p=0.122)$, a reduction from $\beta=0.361(p=0.033)$ in the model without $\mathrm{CP}+\mathrm{HI}$; TP: $\beta=0.294(p=0.067)$, a reduction from $\beta=0.427$ $(p=0.011)$; and NP*TP: $\beta=0.264$, a reduction from $\beta=0.355(p=0.036)$. In models with maternal prenatal smoking, $\mathrm{CP}+\mathrm{HI}$ most strongly predicted time/duration of button pressing (TP): $\beta=0.393$ ( $p=0.016$ ).

\section{Discussion}

Children of mothers who smoked during pregnancy exhibited problems with 'hot' inhibitory control, but not with 'cool' inhibitory control. Furthermore, children of mothers who smoked during pregnancy showed more conduct problems and hyperactivity-inattention than nonexposed children. The reliability of these effects was supported by the fact that dose-response relationships were also observed. The finding that problems particularly appear when cognitive tasks involve the regulation of affect is in line with the well-replicated association between maternal prenatal smoking and behavior regulation problems. In addition, $\mathrm{CP}+\mathrm{HI}$ could account for the effects of prenatal smoking on DeFT-performance. These findings further support the contention that children of mothers who smoked during pregnancy specifically have problems when cognitive task performance involves regulation of affect. Our results indicate the importance of employing a theoretical framework supported by neuro-anatomical and neurobiological data when studying associations between maternal prenatal smoking and children's cognitive and behavioral development.

Although the pattern of results confirmed expectations, it was somewhat surprising that there were no group differences at all for the cool inhibitory control measures, nor were there significant correlations between SA-Dots inhibitory control scores and DeFT inhibitory control scores. Some associations were expected because hot and cool types of EF share common neural structures and neuromodulatory mechanisms, i.e. they are presumed to be part of an 'interactive functional system' (cf. Hongwanishkul et al. 2005), which is in line with the theory stating that cortical processes involving perception and cognition provide representations of the physical and social world to the sub-cortical motivational systems, whilst, in turn, these representations are influenced by appetitive and defensive needs (Derryberry and Rothbart 1997). We did not find evidence supporting such an overlap between hot and cool executive functions, but we cannot rule out that it would become apparent when other measures of cool inhibitory control are used.

Because cognition in children of mothers who smoked during pregnancy has always been studied without a clear theoretical framework, the employment of Zelazo and Müller's model is only a first step towards elucidating the cognitive profile of this population. Further explorations are warranted based on other cognitive models for which clear neuroanatomical evidence exists. It is possible that abnormalities in neuromodulation or neuroanatomical structures supporting hot EF only affect the most demanding cool EFfunctions. The SA-Dots task in the present study had clear inhibitory control demands, but only minimal working memory or flexibility demands, which, particularly in combination with inhibitory control, might represent the most demanding forms of cool EF (e.g. Davidson et al. 2006; Huijbregts et al. 2002b).

Although the present study was the first to use the hot versus cool distinction for examining $\mathrm{EF}$ in children whose 
mothers smoked during pregnancy, studies into ADHD and studies into differences in EF between children with ODD/ $\mathrm{CD}$ and ADHD have employed the same or similar distinctions earlier. In ADHD research significant empirical support has been found for a distinction between delay aversion (which can be considered a form of hot EF) and executive inhibitory control (which can be considered a form of cool EF) (e.g. Luman et al. 2005; Solanto et al. 2001, but see Geurts et al. 2006). Both characterize ADHD, but not necessarily simultaneously. Castellanos et al. (2006) formulated the interesting hypothesis that inattention symptoms may be associated with deficits in cool EF, whereas hyperactivity/impulsivity symptoms reflect hot EF deficits. ADHD-research may however also have limited the conception of hot versus cool EF because hot EF has exclusively been studied in decision making paradigms, mostly (variants of) the Iowa Gambling Task (Bechara et al. 1994). Hot decision-making paradigms are indeed those that involve risks and rewards (Kerr and Zelazo 2004), but the definition of hot EF suggests that the distinction is applicable to most if not all executive functions.

This is important because, within the concept of hot EF, further distinctions may be justified based on which aspect of affect/emotion is involved in performance of a task. For example, frustration and motivation (as measured by for example decision making or varying reward paradigms) are very different emotions with potentially different effects on task performance and different neurobiological correlates. Frustration is more closely related to stress, which, like nicotine, affects ANS/HPA-functioning (stress: Dickerson and Kemeny 2004; Van Goozen et al. 2000; nicotine: Browne et al. 2000; Horne et al. 2004; McDonald et al. 2006; Ramsay et al. 1996). Despite some neuro-anatomical overlap between brain areas active during stress and during motivationally driven situations (e.g. orbitofrontal (OFC-) involvement: Hynes et al. 2006; Rolls 2004), there is no clear-cut evidence for strong ANS/HPA-involvement during decision making tasks (i.e. tasks tapping into motivation). Thus, children of mothers who smoked during pregnancy might particularly demonstrate hot EF-deficits when stress or frustration are involved, but no hot EF deficits when motivation (or reward sensitivity) is involved.

Limitations and recommendations for further studies

Like prenatal smoking, deficient ANS/HPA-functioning is also particularly associated with conduct problems and cooccurring conduct problems and hyperactivity (Herpertz et al. 2001; Waschbusch 2002). Unfortunately the number of participants in the present study was too small to reliably distinguish children with solely hyperactivity, solely conduct problems, and with co-occurring hyperactivity and conduct problems. Larger samples would also be required to investigate whether children with conduct problems present with different types of hot EF-deficits than children with ADHD. The main problem of a small sample size is that it limits the strength of confidence one can have in the generalizability of findings to other samples. That is why this study should be considered exploratory.

Furthermore, it would be desirable for future studies to incorporate a number of control variables in the analysis of hot versus cool executive functions in children of mothers who smoked during pregnancy. There are many different types of variables that could account for associations between maternal prenatal smoking and hot EF-functioning. One important candidate is parental education or IQ, which unlike potential confounders such as alcohol or drug use during pregnancy, quality of parenting, family functioning, family status, mother's age when she had her first child and parental psychopathology, explained associations between maternal prenatal smoking and children's cognitive outcome measures in a number of studies (Breslau et al. 2005; Huijbregts et al. 2006; Trasti et al. 1999). Moreover, associations between maternal prenatal smoking and offspring cognition remained significant in studies where parental education or IQ were not introduced as potential confounders (e.g. Batstra et al. 2003). Despite the fact that these studies investigated associations of prenatal smoking with general cognitive outcome measures such as IQ and academic achievement, which have been reported to correlate significantly with measures of cool but not hot EF (Hongwanishkul et al. 2005), and despite the fact that participants in the present study were all from the same area of Southampton which can be considered relatively uniform regarding socio-economic status (family income, parental education etc.), we have not included a direct control measure for parental IQ or education and can therefore not rule it out as a potentially significant confounder. Moreover, confounders of the association between maternal prenatal smoking and hot EF may be different from those that explain associations between maternal prenatal smoking and cool EF. Another limitation is that, even though our study revealed a clear difference between results on the two inhibitory control tasks, we did not collect children's IQ - or academic achievement measures ourselves. The interpretation of our results would have been stronger if, like Hongwanishkul and colleagues, we would have been able to show that such measures were associated with our cool EF-measure, whilst they would not have had a significant relation with our hot EF-measure.

A final limitation we would like to address concerns the validity of our claim that the Delay Frustration Task measures hot inhibitory control whereas the SA-Dots task measures cool inhibitory control. Whereas the SA-Dots task has been used regularly before to measure inhibition of prepotent 
responding in different clinical populations characterized by deficient inhibitory control (e.g. Huijbregts et al. 2002a; Konrad et al. 2004; Slaats-Willemse et al. 2003), the Delay Frustration Task was originally developed to measure delay aversion (Bitsakou et al. 2006). In the dual pathway model of ADHD, delay aversion is considered a largely autonomous construct (Sonuga-Barke 2002). The advantage of considering this task a measure of delay aversion is that one could then make a stronger case for it being a measure of "hot EF", as such tasks have been used to measure this construct before. Still, the developers of this task recognize that it is likely that the Delay Frustration Task measures inhibitory control (i.e. participants have to stop a "prepotent" or "ongoing" response during the delays) (Bitsakou et al. 2006). Although based on Zelazo and Müller's theory DeFT should be regarded as a hot EF task regardless of whether it is considered a delay aversion or an inhibition task, it might be best for future studies to select tasks which are completely similar apart from an 'affect-' (e.g. frustration) component in one version.

The important message of this study is that two forms of EF can be distinguished which can be classified as hot and cool EF, and that in children of mothers who smoked during pregnancy a discrepancy can be observed between these two. Children whose mothers smoked during pregnancy are particularly at an increased risk of demonstrating (frustration-induced) hot EF-deficits. When these results are confirmed in larger samples that take into account the many potential confounders, intervention programs aimed at child factors could incorporate the training of specific emotion regulation skills in addition to the training of 'cool' EFskills. Ideally, at risk families are identified before or early during pregnancy. Although prenatal smoking occurs in approximately $25 \%$ of pregnant women in Western countries (Breslau et al. 2005; Huijbregts et al. 2006), it has been shown that a family history of antisocial behavior in combination with prenatal smoking seriously compounds the risk of offspring disruptive behavior (Huijbregts et al. in press). Thus, like successful intervention programs aimed at increasing the knowledge about health (including smoking cessation) and care of mothers-to-be such as the Nurse Home Visitation Program (Olds et al. 1999), intervention programs aimed at improving child factors such as their emotion regulation skills should probably be provided to children with a combination of risk factors. When it is confirmed that ANS/HPA abnormalities mediate associations between prenatal smoking and children's disruptive behavior and hot EF, clinical implications might also involve reinstating normal ANS/HPA axis functioning pharmaceutically (Van Goozen et al. 2007), for example by temporarily saturating glucocorticoid receptors. By restoring stress response systems, emotion regulation and related cognitive functioning could be improved, perhaps even more strongly in disruptive children whose mothers smoked during pregnancy than in disruptive children whose mothers did not smoke during pregnancy.

Acknowledgements The authors would like to thank Professor Edmund Sonuga-Barke for allowing the use of the Delay Frustration Task, Peraskevi Bitsakou and Suzannah Helps for providing in-depth information on this task and Luke Phillips for programming it.

\section{References}

Barkley, R. A. (1997). Behavioral inhibition, sustained attention, and executive functions: constructing a unifying theory of ADHD. Psychological Bulletin, 121, 65-94.

Batstra, L., Hadders-Algra, M., \& Neeleman, J. (2003). Effect of antenatal exposure to maternal smoking on behavioural problems and academic achievement in childhood: Prospective evidence from a Dutch birth cohort. Early Human Development, 75, 21-33.

Bechara, A., Damasio, A. R., Damasio, H., \& Anderson, S. W. (1994). Insensitivity to future consequences following damage to human prefrontal cortex. Cognition, 50, 7-15.

Bitsakou, P., Antrop, I., Wiersema, J. R., \& Sonuga-Barke, E. J. (2006). Probing the limits of delay tolerance: Preliminary young adult data from the Delay Frustration Task (DeFT). Journal of Neuroscience Methods, 151, 38-44.

Bourdon, K. H., Goodman, R., Rae, D. S., Simpson, G., \& Koretz, D. S. (2005). The Strengths and difficulties questionnaire: U.S. normative data and psychometric properties. Journal of the American Academy of Child and Adolescent Psychiatry, 44, $557-564$.

Breslau, N., Paneth, N., Lucia, V. C., \& Paneth-Pollak, R. (2005). Maternal smoking during pregnancy and offspring IQ. International Journal of Epidemiology, 34, 1047-1053.

Browne, C. A., Colditz, P. B., \& Dunster, K. R. (2000). Infant autonomic function is altered by maternal smoking during pregnancy. Early Human Development, 59, 209-218.

Button, T. M. M., Thapar, A., \& McGuffin, P. (2005). Relationship between antisocial behaviour, attention-deficit hyperactivity disorder and maternal prenatal smoking. British Journal of Psychiatry, 187, 155-160.

Castellanos, F. X., Sonuga-Barke, E. J. S., Milham, M. P., \& Tannock, R. (2006). Characterizing cognition in ADHD: beyond executive function. Trends in Cognitive Sciences, 10, 117-123.

Castellanos, F. X., \& Tannock, R. (2002). Neuroscience of attentiondeficit/hyperactivity disorder : The search for endophenotypes. Nature Reviews: Neuroscience, 3, 617-628.

Davidson, M. C., Amso, D., Cruess Anderson, L., \& Diamond, A. (2006). Development of cognitive control and executive functions from 4 to 13 years: Evidence from manipulations of memory, inhibition, and task switching. Neuropsychologia, 44, 2037-2078.

Derryberry, G., \& Rothbart, M. K. (1997). Reactive and effortful processes in the organization of temperament. Development and Psychopathology, 1, 339-349.

De Sonneville, L. M. J. (1999). Amsterdam Neuropsychological Tasks: A computer-aided assessment program. In B. P. L. M. Den Brinker, P. J. Beek, A. N. Brand, S. J. Maarse \& L. J. M. Mulder (Eds.), Computers in Psychology, Vol. 6: Cognitive ergonomics, clinical assessment and computer-assisted learning (pp. 187203). Lisse: Swets \& Zeitlinger.

Dickerson, S. S., \& Kemeny, M. E. (2004). Acute stressors and cortisol responses: A theoretical integration and synthesis of laboratory research. Psychological Bulletin, 130, 355-391. 
Fried, P. A., Watkinson, B., \& Gray, R. (1998). Differential effects on cognitive functioning in 9-to-12-year-olds prenatally exposed to cigarettes and marihuana. Neurotoxicology and Teratology, 20, 293-306.

Fried, P. A., Watkinson, B., \& Gray, R. (2003). Differential effects on cognitive functioning in 13- to 16-year-olds prenatally exposed to cigarettes and marihuana. Neurotoxicology and Teratology, 25, 427-436.

Geurts, H. M., Van der Oord, S., \& Crone, E. A. (2006). Hot and cool aspects of cognitive control in children with ADHD: Decisionmaking and inhibition. Journal of Abnormal Child Psychology, $34,813-824$

Goodman, R. (1997). The strengths and difficulties questionnaire: A research note. Journal of Child Psychology and Psychiatry, 38, 581-586.

Goodman, R. (2001). Psychometric properties of the strengths and difficulties questionnaire. Journal of the American Academy of Child and Adolescent Psychiatry, 40, 1337-1345.

Goodman, R., \& Scott, S. (1999). Comparing the strengths and difficulties questionnaire and the child behavior checklist: Is small beautiful? Journal of Abnormal Child Psychology, 27, 17-24.

Henry, B. \& Moffitt, T. E. (1997). Neuropsychological and neuroimaging studies of juvenile delinquency and adult criminal behaviour. In D. D. Stoff, J. Breiling \& J. D. Maser (Eds.), Handbook of antisocial behaviour (pp. 280-288). New York: John Wiley \& Son.

Herpertz, S. C., Wenning, B., Mueller, B., Qunaibi, M., Sass, H., \& Herpertz-Dahlmann, B. (2001). Psychophysiological responses in ADHD boys with and without conduct disorder: Implications for adult antisocial behavior. Journal of the American Academy of Child and Adolescent Psychiatry, 40, 1222-1230.

Hill, J. (2002). Biological, psychological and social processes in the conduct disorders. Journal of Child Psychology and Psychiatry, $43,133-164$

Hongwanishkul, D., Happaney, K. R., Lee, W. S., \& Zelazo, P. D. (2005). Assessment of hot and cool executive function in young children: Age-related changes and individual differences. Developmental Neuropsychology, 28, 617-644.

Horne, R. S. C., Franco, P., Michael Adamson, T., Groswasser, J., \& Kahn, A. (2004). Influences of maternal cigarette smoking on infant arousability. Early Human Development, 79, 49-58.

Huijbregts, S. C. J., De Sonneville, L. M. J., Licht, R., van Spronsen, F. J., Verkerk, O. H., \& Sergeant, J. A. (2002a). Sustained attention and inhibition of cognitive interference in treated phenylketonuria: Associations with concurrent and lifetime phenylalanine concentrations. Neuropsychologia, 40, 7-15.

Huijbregts, S.C.J., De Sonneville, L.M.J., Van Spronsen, F.J., Licht, R., \& Sergeant, J.A. (2002b). The neuropsychological profile of early- and continuously treated phenylketonuria: Orienting, vigilance, and maintenance versus manipulationfunctions of working memory. Neuroscience \& Biobehavioral Reviews, 26, 697-712.

Huijbregts, S. C. J., Séguin, J. R., Zelazo, P. D., Parent, S., Japel, C., \& Tremblay, R. E. (2006). Interrelations between maternal smoking during pregnancy, birth weight and sociodemographic factors in the prediction of early cognitive abilities. Infant and Child Development, 15, 593-607.

Huijbregts, S. C. J., Séguin, J. R., Zoccolillo, M., Boivin, M., \& Tremblay, R. E. (2007). Associations of maternal prenatal smoking with early childhood physical aggression, hyperactivity-impulsivity, and their co-occurrence. Journal of Abnormal Child Psychology, 35, 203-215

Huijbregts, S. C. J., Séguin, J. R., Zoccolillo, M., Boivin, M., \& Tremblay, R. E. Maternal prenatal smoking, parental antisocial behavior, and early childhood physical aggression. Development and Psychopathology (in press).
Hynes, C. A., Baird, A. A., \& Grafton, S. T. (2006). Differential role of the orbital frontal lobe in emotional versus cognitive perspective-taking. Neuropsychologia, 44, 374-383.

Kerr, A., \& Zelazo, P. D. (2004). Development of "hot"executive function: The children's gambling task. Brain \& Cognition, 55, $148-157$.

Klein, T. A., Endrass, T., Kathmann, N., Neumann, J., Von Cramon, D. Y., \& Ullsperger, M. (2007). Neural correlates of error awareness. NeuroImage, 34, 1774-1781.

Konrad, K., Günther, T., Hanisch, C., \& Herpertz-Dahlmann, B. (2004). Differential effects of methylphenidate on attentional functions in children with attention-deficit/hyperactivity disorder. Journal of the American Academy of Child and Adolescent Psychiatry, 43, 191-198.

Kotimaa, A. J., Moilanen, I., Taanila, A., Ebeling, H., Smalley, S. L., McGough, J. J., et al. (2003). Maternal smoking and hyperactivity in 8-year-old children. Journal of the American Academy of Child and Adolescent Psychiatry, 42, 826-833.

Krain, A. L., Wilson, A. M., Arbuckle, R., Castellanos, F. X., \& Milham, M. P. (2006). Distinct neural mechanisms of risk and ambiguity: A meta-analysis of decision-making. NeuroImage, 32, 477-484.

Lau, M. A., Pihl, R. O., \& Peterson, J. B. (1995). Provocation, acute alcohol intoxication, cognitive performance and aggression. Journal of Abnormal Psychology, 104, 150-155.

Law, K. L., Stroud, L. R., LaGasse, L. L., Niaura, R., Liu, J., \& Lester, B. M. (2003). Smoking during pregnancy and newborn neurobehaviour. Pediatrics, 111, 1318-1323.

Luman, M., Oosterlaan, J., \& Sergeant, J. A. (2005). The impact of reinforcement contingencies on $\mathrm{AD} / \mathrm{HD}$ : A review and theoretical appraisal. Clinical Psychology Review, 25, 183-213.

McDonald, S. D., Walker, M., Perkins, S., Beyene, J., Murphy, K., Gibb, W., et al. (2006). The effect of tobacco exposure on the fetal hypothalamic-pituitary-adrenal axis. British Journal of Obstetrics and Gynaecology, 113, 1289-1295.

Mick, E., Biederman, J., Faraone, S. V., Sayer, J., \& Kleinman, S. (2002). Case-control study of attention-deficit hyperactivity disorder and maternal smoking, alcohol use, and drug use during pregnancy. Journal of the American Academy of Child and Adolescent Psychiatry, 41, 378-385.

Moffitt, T. E., Caspi, A., \& Rutter, M. (2005). Strategy for investigating interactions between measured genes and measured environments. Archives of General Psychiatry, 62, 473-481.

Nigg, J. T. (2003). Response inhibition and disruptive behaviors: Toward a multiprocess conception of etiological heterogeneity for ADHD combined type and conduct disorder early-onset type. Annals of the New York Academy of Sciences, 1008, 170-182.

Nigg, J. T., \& Breslau, N. (2007). Prenatal smoking exposure, low birth weight, and disruptive behavior disorders. Journal of the American Academy of Child and Adolescent Psychiatry, 46, 362-369.

Olds, D. L., Henderson, C. R., Kitzman, H. J., Eckenrode, J. J., Cole, R. E., \& Tatelbaum, R. C. (1999). Prenatal and infancy home visitation by nurses: Recent findings. Future of Children, 9, 44-65.

Oosterlaan, J., Logan, G. D., \& Sergeant, J. A. (1998). Response inhibition in $\mathrm{AD} / \mathrm{HD}, \mathrm{CD}$, comorbid $\mathrm{AD} / \mathrm{HD}+\mathrm{CD}$, anxious, and control children: A meta-analysis of studies with the stop task. Journal of Child Psychology and Psychiatry, 39, 411-425.

Oosterlaan, J., Scheres, A., \& Sergeant, J. A. (2005).Which executive functioning deficits are associated with $\mathrm{AD} / \mathrm{HD}, \mathrm{ODD} / \mathrm{CD}$ and comorbid AD/HD + ODD/CD? Journal of Abnormal Child Psychology, 33, 69-85.

Ortiz, J., \& Raine, A. (2004). Heart rate level and antisocial behavior in children and adolescents. Journal of the American Academy of Child and Adolescent Psychiatry, 43, 154-162. 
Pennington, B. F., \& Ozonoff, S. (1996). Executive functions and developmental psychopathology. Journal of Child Psychology and Psychiatry, 37, 51-87.

Pickett, K. E., Rathouz, P. J., Kasza, K., Wakschlag, L. S., \& Wright, R. (2005). Self-reported smoking, cotinine levels, and patterns of smoking in pregnancy. Paediatric and Perinatal Epidemiology, 19, 368-376.

Raine, A. (1993). The psychopathology of crime: Criminal behavior as a clinical disorder. San Diego, CA: Academic Press.

Raine, A. (2002). Annotation: The role of prefrontal deficits, low autonomic arousal, and early health factors in the development of antisocial and aggressive behavior in children. Journal of Child Psychology and Psychiatry, 43, 417-434.

Ramsay, D. S., Bendersky, M. I., \& Lewis, M. (1996). Effect of prenatal alcohol and cigarette exposure on two- and six-monthold infants' adrenocortical reactivity to stress. Journal of Pediatric Psychology, 21, 833-840.

Rolls, E. T. (2004). The functions of the orbitofrontal cortex. Brain and Cognition, 55, 11-29.

Slaats-Willemse, D. S., Swaab-Barneveld, H., De Sonneville, L., Van der Meulen, E., \& Buitelaar, J. (2003). Deficient response inhibition as a cognitive endophenotype of ADHD. Journal of the American Academy of Child and Adolescent Psychiatry, 42, 1242-1248.

Snoek, H., Van Goozen, S. H., Matthys, W., Buitelaar, J. K., \& Van Engeland, H. (2004). Stress responsivity in children with externalizing behavior disorders. Development and Psychopathology, 16, 389-406.

Séguin, J. R., Boulerice, B., Harden, P. W., Tremblay, R. E., \& Pihl, R. O. (1999). Executive functions and physical aggression after controlling for attention deficit hyperactivity disorder, general memory, and IQ. Journal of Child Psychology and Psychiatry, 40, 1197-1208.

Seidler, F. J., Levin, E. D., Lappi, S. E., \& Slotkin, T. A. (1992). Fetal nicotine exposure ablates the ability of postnatal nicotine challenge to release norepinephrine from rat brain regions. Brain Research: Developmental Brain Research, 69, 288-291.

Sergeant, J. A., Geurts, H., Huijbregts, S., Scheres, A., \& Oosterlaan, J. (2003). The top and bottom of ADHD: A neuropsychological perspective. Neuroscience and Biobehavioral Reviews, 27, 583-592.

Slotkin, T. A., MacKillop, E., Rudder, C. L., Ryde, I. T., Tate, C. A., \& Seidler, T. A. (2006). Permanent, sex-selective effects of prenatal or adolescent nicotine exposure, separately or sequentially, in rat brain regions: Indices of cholinergic and serotonergic synaptic function, cell signaling, and neural cell number and size at 6 months of age. Neuropsychopharmacology, 31, 1-16.

Solanto, M. V., Abikoff, H., Sonuga-Barke, E., Schachar, R., Logan, G. D., Wigal, T., et al. (2001). The ecological validity of delay aversion and response inhibition as measures of impulsivity in $\mathrm{AD} / \mathrm{HD}$ : A supplement to the NIMH Multimodal Treatment Study of AD/HD. Journal of Abnormal Child Psychology, 29, 215-228.

Sonuga-Barke, E. J. (2002). Psychological heterogeneity in AD/HD: A dual pathway model of behaviour and cognition. Behavioural Brain Research, 130, 29-36.

Trasti, N., Vik, T., Jakobsen, G., \& Bakketeig, L. S. (1999). Smoking in pregnancy and children's mental and motor development at age 1 and 5 years. Early Human Development, 55, $137-147$.

Van Goozen, S. H., Cohen-Kettenis, P. T., Snoek, H., Matthys, W., Swaab-Barneveld, H., \& Van Engeland, H. (2004). Executive functioning in children: A comparison of hospitalised ODD and ODD/ADHD children and normal controls. Journal of Child Psychology and Psychiatry, 45, 284-292.

Van Goozen, S. H. M., Fairchild, G., Snoek, H., \& Harold, G. T. (2007). The evidence for a neurobiological model of childhood antisocial behavior. Psychological Bulletin, 133, 149-182.

Van Goozen, S. H. M., Matthys, W., Cohen-Kettenis, P. T., Buitelaar, J. K., \& Van Engeland, H. (2000). Hypothalamic-pituitaryadrenal axis and autonomic nervous system activity in disruptive children and matched controls. Journal of the American Academy of Child and Adolescent Psychiatry, 39, 1438-1445.

Wakschlag, L. S., Pickett, K. E., Kasza, K. E., \& Loeber, R. (2006). Is prenatal smoking associated with a developmental pattern of conduct problems in young boys? Journal of the American Academy of Child and Adolescent Psychiatry, 45, 461-467.

Waschbusch, D. A. (2002). A meta-analytic examination of comorbid hyperactive/impulsive/inattention problems and conduct problems. Psychological Bulletin, 128, 118-150.

Wiersema, J. R., Van der Meere, J.J., \& Roeyers, H. (2007). Developmental changes in error monitoring: An event-related potential study. Neuropsychologia, 45, 1649-1657.

Wilcutt, E. G., Doyle, A. E., Nigg, J. T., Faraone, S. V., \& Pennington, B. F. (2005). Validity of the executive function theory of attention deficit/hyperactivity disorder: A meta-analytic review. Biological Psychiatry, 57, 1336-1346.

Zelazo, P. D., Carter, A., Reznick, J. S., \& Frye, D. (1997). Early development of executive function: A problem-solving framework. Review of General Psychology, 1, 198-226.

Zelazo, P. D., \& Müller, U. (2002). Executive function in typical and atypical development. In U. Goswami (Ed.), Handbook of childhood cognitive development (pp. 445-469), Oxford, England: Blackwell.

Zuckerman, M. (1979). Sensation seeking: Beyond the optimum level of arousal. Hillsdale, NJ: Erlbaum. 